\title{
Mites Associated with Water Weeds in Egypt
}

\author{
A. K., Nasr; Magda, M. Abou-Elela and Kh. M. A. Saleh \\ Pests \& Plant Protection Dept., National Research Centre, Dokki, Cairo, Egypt.
}

\begin{abstract}
A survey on mites associated with water weeds was conducted at different areas. Random samples were collected from different locations. Nineteen families belonging to three sub-orders were found. Sub-order Gamasida included eight families namely Parasitidae, Digamasellidae, Ologamasidae, Ascidae, Ameroseiidae, Phytoseiidae, Macrochelidae and Laelapidae, Sub-order Actinedida included five families namely Bdellidae, Cunaxidae, Stigmaetidae, Teternychidae and Eriophyidae and sub-order Oribatida comprised six families namely Hypochthoniidae, Lohmannidae, Oppidae, Hydrozetidae, Oribatulidae and Galumnidae.
\end{abstract}

Key Words: Water weeds, Acari, Gamasida, Actinedida, Oribatida.

\section{INTRODUCTION}

Weeds decrease crop yields, increase coasts of production and reduce the quality of crop and livestock products (Buchholtz, 1967). Water hyacinth, Eichhornia crassipes (Mart.) is a perennial aquatic floating weed which grows and multiplies in fresh water of reservoirs, dams, rivers and drainage canals, lagoons and lakes. It may be considered as the worst aquatic weed invading water canals and reservoirs in tropical, subtropical and warm countries allover the world (Viet-Meyer, 1975; Holm et al., 1977). In Africa, it was first recorded in the Sudan in 1955 (Beshir and Bennett, 1985). Although E. crasspies was recorded in Egypt in 1980 , it only became a serious threat in the River Nile in 1960.

The rapid spread of E. crasspies in the Nile Delta is a normal consequence to the development of river control schemes, particularly the Aswan High Dam (Batanouny et al., 1984).

Weeds usually act as dwellings for many mites and insects. The arthropods collected from water hyacinth in its native range of distribution constitute a list of about 43 different species (Perkins, 1974a). Half of them hardly cause noticeable damage or have a wide range of food plants.

Research done by Tuttle et. al., (1977) indicated that only seven species belonging to family Tetranychidae were collected from the water weed Convolvulu arvensis !

Cromroy and Reinert (1981) reported three mite species belonging to the families Tarsonemidae and Eriophyidae collected from the water weeds. In 2003, $\mathrm{Haq}$ and Sumangala showed that a survey of mite fauna associated with water hyacinth yielded 21 species belonging to two orders (Actinedida and Oribatida) and nine families. Among these, three species of Tetranychidae, namely Eutetranychus orientalis
(Klein) Tetranychus ludeni Zacher, and Oligonychus biharensis (Hirst), and one species of Galuminiidae, Orthogalumna terebrantis Wallwork has been demonstrated to cause feeding damage to the host plant.

Orthogalumna terebrantis is one of a few mites causing phytodamages to water hyacinth (Bennett, 1968; 1970; 1974; Del Fosse et. al., 1975). Perkins (1974b) found that mites could enter pseudolamine (false leaves) to feed.

In Egypt very little work concerning water weeds was done. Therefore, the present work aims to throw light on the incidence of different phytophagous, predacious and saprophagous mite species associated with water weeds.

\section{MATERIALS AND METHODS}

\section{A. Sampling extraction and identification of various mites associated with water weeds. \\ 1. Sampling:}

Samples of water weeds with apparent damage symptoms were collected from some localities, throughout three years during $(2004-2006)$ at certain Governorates. Localities studied were: Qalubyia, Monofiya, Dymiat, Bani - Sweef and Assiut.

\section{Extraction:}

Owing to the samples consisting entirely of organic matter, a heat desiccation method of extraction based on the Tullgren funnel was used in preference to a flotation method. The extracted mites were received in Petri dish with a piece of moistened cotton.

\section{b. Identification of mites:}

The generic concepts of most mesostignatic mites followed Lindquist \& Evans (1965) and de Moreas et al., (2004). The generic concepts of oribated mites followed Balough (1972). 
Table (1): Mite species associated with water weeds, during March 2004 to December 2006

\begin{tabular}{cll}
\hline Family & \multicolumn{1}{c}{ Species } & \multicolumn{1}{c}{ Weeds/Habitat } \\
\hline Parasitidae & Parasitus zaheri Hafez \& Nasr & $\begin{array}{l}\text { Eichhornia crassipes (Mart.) (leaves) } \\
\text { Conzya dioscoridis (L.) } \\
\text { Parasitus badrii Hafez \& Nasr }\end{array}$ \\
& (leaves)
\end{tabular}

\begin{tabular}{lll}
\hline Digamasellidae & Dendrolaelaps aegypticus Metwally \& & E. crassipes (Mart.) (root) \\
& Mersal & Conyza dioscoridis (L.) (leaves). \\
& Arondo donax L. (leaves) \\
& Ceratophyllum demersum L. (leaves)
\end{tabular}

Ologamasidae Garnasiphis pulchellus (Berlese)

E. crassipes

Gamasiphis aegypticus Nasr \& Afifi (roots)

Silybium marianum (L) (leaves)

\begin{tabular}{|c|c|c|c|}
\hline \multirow[b]{2}{*}{ Ascidae } & & & El-Bagoor, Santres \\
\hline & $\begin{array}{l}\text { Lasioseius lindquisti } \\
\text { Nasr and Abou Awad } \\
\text { Lasioseius athiasae Nawar and Nasr } \\
\text { Lasioseius zaheri Nasr } \\
\text { Lasioseius africanus Nasr } \\
\text { Cheiroseius nepalensis Evans and Hyatt } \\
\text { Cheiroseius egypticus Hussein and } \\
\text { Mazen }\end{array}$ & $\begin{array}{l}\text { E. crassipes (M.) (roots) } \\
\text { C. dioscoridis (L.) (leaves) } \\
\text { A. donax L. (leaves) } \\
\text { A. donax L. (leaves) } \\
\text { A. donax L. (lcaves) } \\
\text { A. donax L. (leaves) }\end{array}$ & $\begin{array}{l}\text { Banha. Met-Elramla } \\
\text { Tokh, Alamar } \\
\text { Kaluob, Bahada } \\
\text { Mostorud, Khosos } \\
\text { El-Badary, Afadra } \\
\text { Manfalout. Banyady } \\
\text { Kewesna, Mostay } \\
\text { Sheben El-Kom, Astobary } \\
\text { Bosh, Der-Elnahia }\end{array}$ \\
\hline Ameroseiidae & $\begin{array}{l}\text { Cheiroseiulus crassipes Ramadan } \\
\text { Proctolaelaps orientalis Nasr } \\
\text { Proctolaelaps aegyptiaca Nasr } \\
\text { Ameroseius agypticus } \mathrm{El-Badry,} \mathrm{Nasr} \mathrm{\&} \\
\text { Hafez. }\end{array}$ & $\begin{array}{l}\text { E. crassipes (M.) (leaves) } \\
\text { A. donax L. (leaves) } \\
\text { C. demersum L. (leaves) } \\
\text { C. demersum L.(leaves) }\end{array}$ & $\begin{array}{l}\text { Tokh, Alamar } \\
\text { Bosh, Der-Elnahia } \\
\text { El- Badary. Afadra }\end{array}$ \\
\hline Phytoseiidae & $\begin{array}{l}\text { Neoseinulus barkeri (Hughes) } \\
\text { Amblyseius zaheri Yousef \& Fl- } \\
\text { Borolossy } \\
\text { Euseius yousefi (Zaher \& El-Borolossy) } \\
\text { Proprioseiopsis lindquisti (Schuster \& } \\
\text { Pritchard) } \\
\text { Typhlodromips swirskii (Athias-I Ienriot) } \\
\text { Euseins scutalis ( } \Lambda \text { thias \& Henriot) } \\
\text { Typhlodromus transvaalensis Nesbitl } \\
\text { Typhlodromus negevi Swirski \& Amitai } \\
\text { Phytoseius finitimus Ribaga }\end{array}$ & $\begin{array}{l}\text { E. crassipes (M.) (leaves) } \\
\text { C. dioscoridis (L.) (leaves) } \\
\text { A. donax L. (leaves) } \\
\text { S. marianum (L.) (leaves) } \\
\text { C. dactulon (I..) (leaves) } \\
\text { Oxalis corniculata L. (leaves) } \\
\text { O. corniculata L. (leaves) } \\
\text { O. corniculata I.. (leaves) } \\
\text { O. conniculata L.. (leaves) }\end{array}$ & $\begin{array}{l}\text { Banha. Met-Elramla } \\
\text { Tokh, Alamar } \\
\text { Sheben El-Kom. Astobary } \\
\text { Kewesna, Mostay } \\
\text { Manfalout. Banyady } \\
\text { Dayroot. Sanabo } \\
\text { Farscor, Kafr-Elarab } \\
\text { Bosh. Der-LInahia }\end{array}$ \\
\hline Macrochelidae & Macrocheles sp. & E. crassipes (M.) (roots) & $\begin{array}{l}\text { Banha. Met-Elramla } \\
\text { Manfalout, Banyady }\end{array}$ \\
\hline Laelapidae & $\begin{array}{l}\text { Hypoaspis reficulatus } \\
\text { IJusscin \& Mazen } \\
\text { Ololuclaps chanti Hussein \& Mazen }\end{array}$ & $\begin{array}{l}\text { E. crassipes (M.) (roots) } \\
\text { C. dioscoridis (L.) (roots) } \\
\text { A. donax L. (roots) } \\
\text { S. marianum (I.) (roots) } \\
\text { (. demersum L. (roots) }\end{array}$ & $\begin{array}{l}\text { Tokh. Alamir } \\
\text { Kewesna. Mostay } \\
\text { 11-Bagoor. Sintres } \\
\text { Bosh. Der-Einahia } \\
\text { El-wasta. El-Dohya } \\
\text { El-Badary. Afadra }\end{array}$ \\
\hline Cunaxidae & (unctuer setirustris (Hermam) & $\begin{array}{l}\text { E. crassipes (M.) (leaves) } \\
\text { C. dioscoridis. (L.) (lcaves) } \\
\text { A. donax L (leaves) }\end{array}$ & $\begin{array}{l}\text { Tokh. Alamar } \\
\text { Kewcsna. Mostay } \\
\text { Manfaiout. Banyady }\end{array}$ \\
\hline Bdellidae & $\begin{array}{l}\text { Spinibdella bifuicata (Atyeo) } \\
\text { Cyta latirostris (Hermann) }\end{array}$ & $\begin{array}{l}\text { E. crassipes (M.) (leaves) } \\
\text { C. dioscoridis (I..) (leaves) } \\
\text { A. donax L. (leaves) } \\
\text { C. dectylon (L.) (leaves) }\end{array}$ & $\begin{array}{l}\text { Banha. Met-litamla } \\
\text { Tokh. Alamar } \\
\text { Mostorud. Khosos } \\
\text { Berket F-Sabi. Iloren } \\
\text { Sheben El-Kom. Astohary } \\
\text { Manfalout. Banyady } \\
\text { G-Badary. Ahicira }\end{array}$ \\
\hline Stigmacidae & Agistemus exsertus Gonzales & $\begin{array}{l}\text { E. crassipes (M.) } \\
\text { (leaves) } \\
\text { C. dioscoridis (I.) (leaves) } \\
\text { A. donax L. (leaves) } \\
\text { O. cornculata (leaves) }\end{array}$ & 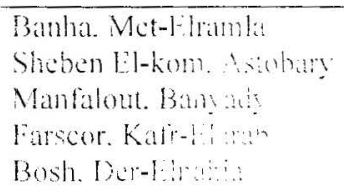 \\
\hline
\end{tabular}


Continued: Table (1)

\begin{tabular}{clll}
\hline Family & \multicolumn{1}{c}{ Species } & \multicolumn{1}{c}{ Weeds/Habitat } & \multicolumn{1}{c}{ Locality } \\
\hline Tetranychidae & Tetranychus urticae Koch & E. crassipes (M.) (leaves) & Banha, Met-Elramla \\
& Eutetranychus pyri (Attiah) & C. dioscoridis (L.) (leaves) & Mostorud, Khosos \\
& Bryobia praetiosa Koch & C. dactylon (L.) (leaves) & Tokh. Alamar \\
& Oligonychus krantzi Zaher, Gomaa \& & Cyperus difformis (L.) (leaves) & Sheben El-Kom, Astobary \\
& El-Enany & O. corniculata L. (leaves) & Berket El Sabi, Horen \\
& & & Farscor. Kalr-Elarab \\
& & & Bosh, Der-Elnahia \\
& & El-Fashn. Bany Saleh \\
& & Manfalout. Banyady
\end{tabular}

\begin{tabular}{lll}
\hline Eriophyidae & $\begin{array}{l}\text { Aceria dioscoridis (Soliman \& Abou-- } \\
\text { Awad) }\end{array}$ & Conyze dioscoridis (L.) (leaves) \\
& Eriophyes cynodoniesis (Sayed) & C. dactylon (L.) (leaves)
\end{tabular}

Banha, Met-Elramla

Tokh, Alamar

Manfalout. Banyady

El-Badary, Afadra

Farscor, Kafr-Elarab

Berket El- Sabi.Horen

Bosh, Der-Elnahia

\begin{tabular}{|c|c|c|c|}
\hline Hypochthoniidae & Hypochthonius sp. & E. crassipes (M.) (root) & Manfalout, Banyady \\
\hline Lohmanniidae & $\begin{array}{l}\text { Lohmannia aegypticus } \\
\text { El-Badry \& Nasr }\end{array}$ & E. crassipes (M.) (roots) & $\begin{array}{l}\text { Tokh, Alamar } \\
\text { El-Badary, Afadra } \\
\text { Sheben Fl-Kom. Astobary }\end{array}$ \\
\hline Oppiidae & $\begin{array}{l}\text { Oppia sitnikovae (Shereef) } \\
\text { Multioppia wilsoni Aoki } \\
\text { Oppiella nilituca Popp }\end{array}$ & $\begin{array}{l}\text { E. crassipes (M.) } \\
\text { (roots) }\end{array}$ & $\begin{array}{l}\text { Banha, Met-Elramla } \\
\text { El-Badary, Afadra } \\
\text { Kewesna, Mostay }\end{array}$ \\
\hline Hydrozetidae & Hydrozetes sp. & E. crassipes (M.) (roots) & $\begin{array}{l}\text { Manfalout. Banyady } \\
\text { Kewesna, Mostay } \\
\text { Sheben El-Kom, Astobary } \\
\text { El-Wasta. El-Dahya } \\
\text { Farscor, Kafr-Elarab }\end{array}$ \\
\hline Galumnidae & Galumna flebillifera Hammer & E. crassipes (M.) (roots) & $\begin{array}{l}\text { Banha, Met-Elramla } \\
\text { Bosh, Der-Elnahia } \\
\text { El-Badary, Afadra }\end{array}$ \\
\hline
\end{tabular}

\begin{tabular}{llll}
\hline Oribatulidae & Zygoribatula tamevai El-Badry \& Nasr & E. crassipes (M.)(roots) & Banha, Met-Elramla \\
& Scheloribates laevigatus (koch) & Ceratophyllum demersum L. & El-Bagoor, Santres \\
& Scheloribates zahari Yousef \& Nasr & (roots) & Farscor, Kafr-Elarab \\
& & Lemn gibba L. (roots) & Bosh. Der-Elnahia \\
& & El-Badary, $\Lambda$ fadra \\
\hline
\end{tabular}

\section{RESULTS AND DISCUSSION}

\section{A. Mites associated with water weeds:}

Forty seven mite species belonging to three sub orders and 19 families were collected from various water weeds. The materials collected were namely Eichhornia crassipes, (Mart.) Conzya dioscoridis (L.) Arondo donax L. Lemm gibba L. Oxalis corniculata L. Cynodon dactylon (L.) and Cyperus difforims (L.). The localities of collected samples were from Qalubyia, Monofiya, Dymiat, Bani-Sweef and Asuot Governorates. Mites of sub order Gamasida included eight families, namely Parasitidae, Digamasellidae, Ologamasidae, Ascidae, Ameroseiidae, Phytoseiidae, Macrochelidae, and Laelapidae.

The suborder Actinedida included five families, namely Bdellidae, Cunaxidae. Stigmaeidae, Tetranychidae and Eriophyidae. The suborder Oribatida was represented by six families, namely
Hypochthoniidae, Lohmanniidae, Oppiidae, Hydrozetidae, Oribatulidae and Galumnidae.

Mites collected during the present study at different localities in Egypt are shown in Table, 1.

Eichhornia crassipes (Mart.) was the commen water weed in the survey. The rich fauna recorded herein on E. crassipes and other water weeds could be due to the fact that the sampling covered the whole plant (leaves and roots) comparing with studies where only leaves were examined.

Members of the family Ascidae represented by nine species on roots and leaves, while Phytoseiidae including nine species on leaves only, are the most dominant groups in this study. Feeding habits of both families are different. since phytoseiid mites were found on leaves only, whereas ascid mites were found on roots and leaves of water weeds. 


\section{REFERENCES}

Balough, J. 1972. The oribatid genera of the world. Akad. Kiado. Budapest: 1-188.

Batanouny, K. H.; El-Fiky, A. M. and Thyagarajan, G. 1984. Water hyacinth in Egypt: distribution and problem magnitude, Proceedings of The International Conference on Water Hyacinth.

Bennett, F. D. 1968. Infestation of insects attacking water hyacinth in Florida. British Honduras and Jamica, 1968. C. I. B. C.Rep. 9 pp.

Bennett, F. D. 1970. Insects attacking water hyacinth in the West India, British Honduras and the U. S. A. Hyacinth contr. J. 5: 10-13.

Bennett, F. D. 1974. Biological control. Pages 99106 in D. S. Mitchell, ed Aquatic Vegetation and its use and control. UNESCO, Paris.

Beshir, M. O. and Bennett, F. D. 1985. Biological control of water hyacinth on the while Nile, Sudan. Proceedings of the VI. Internation Symposium on Biological control of weeds.

Buchholtz, K. P. 1967. Report of the terminology committee of the weed science society of America, Weeds Res., 15: 388-389.

Cromory, H. L. and Reinert, J. A. 1981. The pest mite species of grass in Florida. proceedings of Fla. Turf Grass Assoc., 29: 88-91.

de Moreas, G. I.; McMurtry, J. A.; Denemark, A. H. and Campos, C. B. 2004. A revised catalog of the mite family Phytoseiidae. Zootaxa
434, $494 \mathrm{pp}$.

Del Fosse, E. S.; Cromory, H. L. and Habeck, D. H. 1975. Determination of the feeding mechanism of the water hyacinth mite. Hyacinth contr. J. 13: 53-55.

Haq, M. A. and Sumangala, K. 2003. Acarine regulators of water hyacinth in kerala (India). Exp. Appl. Acarol., 29 (1-2) 27-33.

Holm, L. G.; Pluchnet, D. L.; Pancho, J. V. and Heberger, J. P. 1977. The worlds worst weeds distribution and biology. Univ. Press. Hawaii Honolulu, $609 \mathrm{pp}$.

Lindquist, E. E. and Evans, G. O. 1965. Taxonomic concept in the Ascidae, with a modified setae nomenclature for the idiosoma of the Gamasina (Acarina :Mesostigmata). Memoirs of the Entom. Soc. of Canada, 47: 1-64.

Perkins, B. D. 1974a. Preliminary studies on a strain of the water hyacinth mite from Argentina. Pages 179-184 in Proc. Internet. Symp. On boil. Contr. Of weeds, 4-7 October, 1971, Roma, Italy.

Perkins, B. D. 1974b. Arthropods that stress water hyacinth, PANS, 20 (3): 304-314.

Tuttle, D. M.; Baker, E. W. and Sales, F. M. 1977. Spider mites (Tetranychidae: Acarina) of the state of Ceara, Brazil-Int. Z. Acrol., 3 (1): 1-8.

Viet-Meyer, N. D. 1975. The beautiful blue devil. The American Museum of Natural History. An IPPC papers reprint from National History Magazine. $5 \mathrm{pp}$. 